\title{
Theory of Electrical Conduction Through a Molecule
}

\author{
ELDON G. EMBERLY ${ }^{a}$ AND GEORGE KIRCZENOW \\ Department of Physics, Simon Fraser University, Burnaby, British Columbia, Canada \\ V5A 1 S6
}

\begin{abstract}
We present a theoretical study of electronic transport through a 1,4 benzene-dithiolate (BDT) ion chemically bonded to two gold leads. The model consists of a single BDT attached to two Au clusters, which are in turn attached to ideal one-dimensional leads. Extended Hückel theory is used to model the electronic structure and the Lippmann-Schwinger equation is solved for the lead-to-lead transmission. The current-voltage characteristics of the system are calculated using the finite temperature, finite voltage Landauer formula. Different atomic configurations of the Au clusters are examined including the effects of thermally induced positional disorder of the $\mathrm{Au}$ atoms. We find that while in some cases there is conduction via states derived from the BDT HOMO and LUMO levels, conduction due to levels derived from hybrid Au cluster-BDT states often predominate. The current-voltage characteristics are found to be sensitive to the specific configuration of the Au clusters, BDT and ideal leads.
\end{abstract}

\section{INTRODUCTION}

There has been renewed interest in studying the electronic transport properties of molecular systems in recent years. The novel ideas first envisioned by Ratner and Aviram ${ }^{1}$ are now being realized and characterized experimentally, ${ }^{2-7}$ as new molecular devices are being fabricated. Theoretical ideas and calculations are also emerging motivated in part by the experimental findings. ${ }^{8-15}$

One of the devices is a molecular wire which may be studied by passing current through a molecule attached to two metallic leads. A molecule of current interest for use as a molecular wire is 1,4 benzene-dithiol, which when attached to two gold leads becomes 1,4 benzene-dithiolate (BDT) ${ }^{2,10}$ It is a good candidate since the benzene ring offers delocalized states in the form of $\pi$ bonds which would seem to be beneficial to electron transport. The sulphur end groups also allow for the desired functionality of bonding readily to the gold leads. Many other candidates exist, but the transport properties of this molecule have been measured experimentally ${ }^{2}$ and thus data are available for comparison with calculations.

Many questions arise when considering the microscopic nature of the lead-molecule-lead system: What is the atomic configuration of the leads, and how does the molecule bond to the leads' surfaces? Should the coupling between the molecule and leads be regarded as strong or weak? What role does the structure and chemistry of the system play in transport?

${ }^{a}$ Address for telecommunication: phone, 604/291-3480; fax, 604/291-3592; e-mail: eemberly @ sfu.ca 
To address the issue of the microscopics, specifically the coupling between the molecule and metallic leads, we have modeled the system in a somewhat different fashion from that of previous studies. To complement previous work that has modeled molecules connected to semi-infinite macroscopic leads, we have chosen to represent the leads as finite gold clusters to which ideal leads are attached. The clusters bind to the BDT, and we treat the complex consisting of the BDT, and clusters as an integral chemical entity in order to address the chemical nature of the junctions between the molecule and leads as well as is possible at the level of our calculations. It seems reasonable that the binding of the metallic system to the molecule strongly affects the electronic nature of the molecule. We find that conduction through the molecule is not necessarily controlled by the highest occupied molecular orbital (HOMO) and lowest unoccupied molecular orbital (LUMO) when the molecule is bonded to leads. Thus it is important to focus on the states of the coupled molecularmetallic system rather than on those of the molecular subsystem alone. Because of the sensitivity to the nature of the coupling, the microscopic details of the structure have a strong influence on the resulting transport.

In Section II, we describe the scattering and transport theory used in this work. It is based on a direct numerical solution of the Lippmann-Schwinger equation. The evolution of the energy states of the system when Au clusters are attached to the BDT is discussed in Section III. The calculated transmission and conductance for various BDT-cluster systems is presented in Section IV. Finally, the effect of elastic scattering of electrons by thermal atomic vibrations in the Au clusters is considered in Section V.

\section{THEORY}

The transport of electrons through a molecule connected to two metallic leads can be modeled in many different ways. At its simplest level, this is a tunneling problem where an electron incident from the source with energy $E$ has a probability $T(E)$ of being transmitted through the structure to the drain. By calculating this transmission probability for a range of energies around the Fermi energy, $\epsilon_{F}$ of the lead, the finite temperature, finite voltage, Landauer formula can be used to calculate the transmitted current

$$
I(V)=\frac{2 e}{h} \int_{-\infty}^{\infty} d E T(E)\left(\frac{1}{\exp \left[\left(E-\mu_{s}\right) / k T\right]+1}-\frac{1}{\exp \left[\left(E-\mu_{d}\right) / k T\right]+1}\right)
$$

The two chemical potentials $\mu_{s}$ and $\mu_{d}$, refer to the source and drain, respectively. They are defined to be, $\mu_{s}=\epsilon_{F}+e V / 2$ and $\mu_{d}=\epsilon_{F}-e V / 2$, where $V$ is the sourcedrain bias voltage.

We calculate the transmission probability $T(E)$ starting from the LippmannSchwinger (LS) equation,

$$
|\Psi\rangle=\left|\Phi_{0}\right\rangle+G_{0} W|\Psi\rangle
$$

Here, both $\left|\Phi_{0}\right\rangle$, the state of the incident electron, and $G_{0}$, the free propagator, refer to the unperturbed system. We take the unperturbed system to consist of three non- 


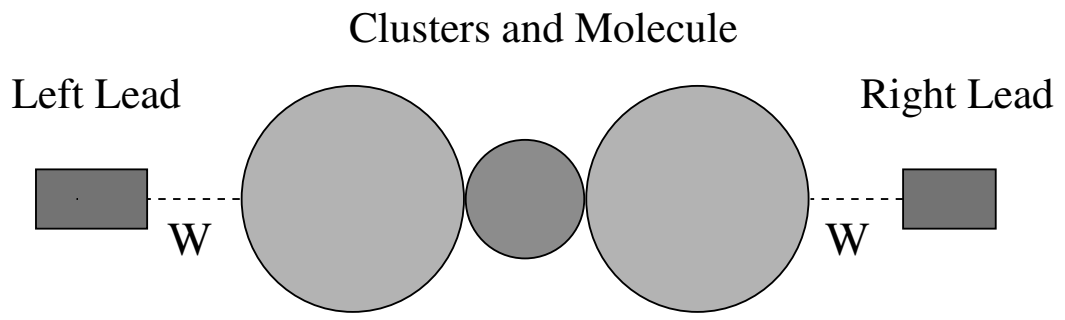

FIGURE 1. Schematic diagram of the three noninteracting systems: left lead, CMC, and right lead. The systems are coupled by the potential $W$.

interacting subsystems. These are the left lead, the right lead, and the clusters+molecule (CMC), as illustrated in FIGURE 1. These noninteracting subsystems are then coupled by an interaction Hamiltonian, $W$, which connects the free propagator $G_{0}$ between the subsystems and transforms the incident one electron state, $\left|\Phi_{0}\right\rangle$, into the scattering state $|\Psi\rangle$.

Each of the state vectors, $\left|\Phi_{0}\right\rangle$ and $|\Psi\rangle$, are represented using LCAO, where the basis is composed of the atomic orbitals on the CMC and the leads. The ideal leads attached to the CMC are modeled as semi-infinite 1-D chains of atoms with one orbital per site, and only nearest neighbor hopping. The incoming boundary conditions for $\left|\Phi_{0}\right\rangle$ are such that it is an incoming Bloch wave in the left lead, and has a value of 0 on the CMC and right lead systems. This allows for an exact analytical evaluation for the free propagator on the leads. The free propagator for the CMC is calculated using its energy eigenstates. These can be found via different quantum chemistry methods, but for our calculation, they were determined by using simple extended Hückel. Because initially the three subsystems are not coupled to each other, the free propagator connecting sites between subsystems is zero.

The matrix elements for the coupling potential $W$ are defined to be non-zero only between the lead sites adjacent to the CMC and sites on the CMC. We approximate them by using extended Hückel to calculate the Hückel matrix elements between the single lead orbital and all orbitals on the CMC. Thus the coupling $W$ depends on where the lead site is placed with respect to the CMC, and also on the nature of its atomic orbital.

We solve the LS equation, Eq. (2), for $|\Psi\rangle$ on the lead sites adjacent to the CMC and also for the sites on the CMC. The transmission, $T(E)$ is then just the square modulus of $|\Psi\rangle$ on the site of the right lead adjacent to the CMC.

\section{ENERGY LEVEL SCHEMES}

A possible controlling factor in understanding molecular wires is the nature of the coupling of the continuum of energy states in the lead to the discrete energy levels of the molecular system. We believe it to be important to study how the energy levels of the molecule change when placed in contact with the metallic leads. The nature of these changes will be discussed below for different configurations of the contacts and the BDT molecule. 


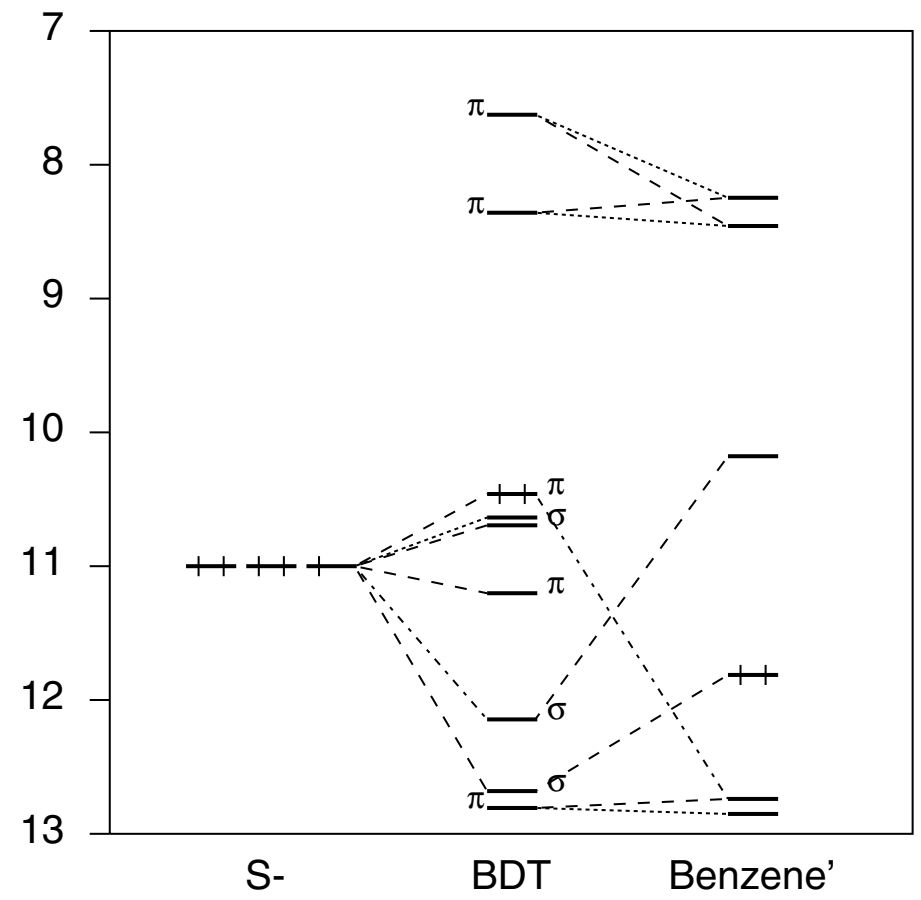

FIGURE 2. Energy-level diagram for BDT ion. Left diagram shows sulphur ion (S) p orbitals. Not shown is the sulphur S level, which is lower in energy. Right diagram is for benzene with the 1,4 Hs removed. The dashed lines connecting the diagrams indicate where the levels of a given segment get mixed into the BDT ion.

When 1,4 benzene-dithiol reacts with gold, it has been experimentally shown ${ }^{17}$ that it loses a pair of $\mathrm{H}$ ions to become BDT. The $\mathrm{H}$ ions react with the gold by capturing a valence electron. In FIGURE 2, the energy level diagram of BDT is shown. To its right in the figure are the energy levels for benzene with the Hs at positions 1 and 4 removed (labeled Benzene'). On its left are shown the energy levels for the sulphur end groups of the BDT ion. These were calculated using extended Hückel. From a calculation for benzene (not benzene') the HOMO is at $-12.814 \mathrm{eV}$ with a LUMO of $-8.248 \mathrm{eV}$. Both of these levels describe $\pi$ bonds on the $\mathrm{C}$ ring, and it is shown on the diagram where the corresponding benzene' levels mix into the BDT. The HOMO-LUMO gap shrinks for BDT with the HOMO now at $-10.470 \mathrm{eV}$, but with almost the same LUMO of $-8.247 \mathrm{eV}$. The character of the HOMO is that of $\mathrm{C} \pi$ around the ring with some $\mathrm{S} \pi$ content. The other levels in the BDT spectrum between the benzene HOMO-LUMO also have $\mathrm{S}$ character, and as marked on the diagram, also contain $\mathrm{C} \pi$ and $\mathrm{C} \sigma$ bonding states. The $\sigma$ states are due to the two states in the benzene' spectrum that occur within the traditional HOMO-LUMO gap of benzene. It will be seen that these levels are significantly influenced when the $S$ is bonded to the Au clusters. 

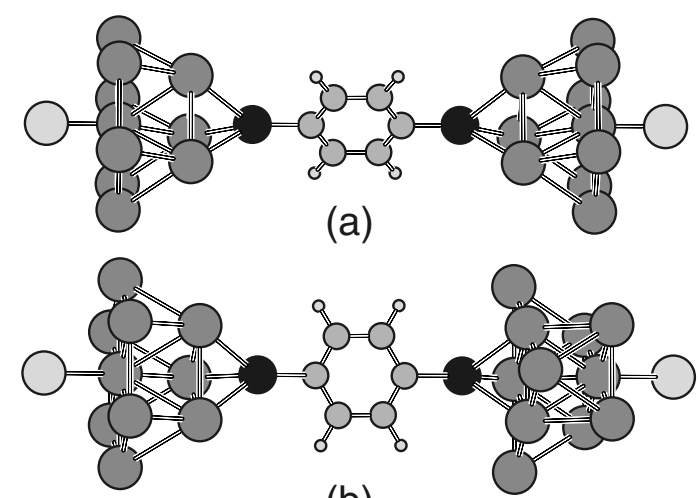

(b)
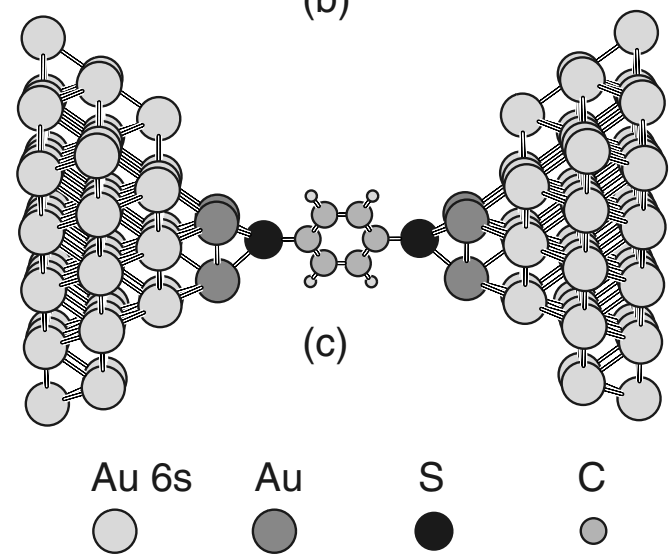

Figure 3. Atomic diagrams of CMC systems. (a) $10 \mathrm{Au}$ (111) clusters attached to BDT. (b) $52 \mathrm{Au}$ (111) clusters attached to BDT. (c) 10/11 Au (111) clusters attached to BDT. In (b), the two lead atoms that bond to the left and right clusters are not shown.

No studies as yet have been done to characterize the nature of the interface between the sulfur and the gold nanowire. Recent studies of gold nanowires and break junctions of macroscopic gold wires ${ }^{18,19}$ have shown them to be different from bulk gold; the region between two gold contacts is composed of filament structures. The reconstructions of the Au atoms for a nanobridge tend to form hcp structures. Though neither of these reports may characterize the exact nature of the interface, it seems reasonable to model the leads with different geometrical configurations that are consistent with the above findings for non-bulk gold. The cluster contacts to be considered are shown in FIGURE 3. In all the clusters, the gold atoms have been arranged in an ideal fcc (111) configuration with the lattice parameter of bulk gold. It has been assumed that the Ss of the BDT each bond to a triangle of Au atoms. An SCF calculation by Sellers ${ }^{16}$ has shown this to be energetically favorable for an alkanethiolate. The perpendicular distance of the S from the Autriangle has been chosen to be 1.9 angstroms. The other important bond is the $\mathrm{S}-\mathrm{C}$ bond, which has been chosen to be 1.795 angstroms. 
(a)

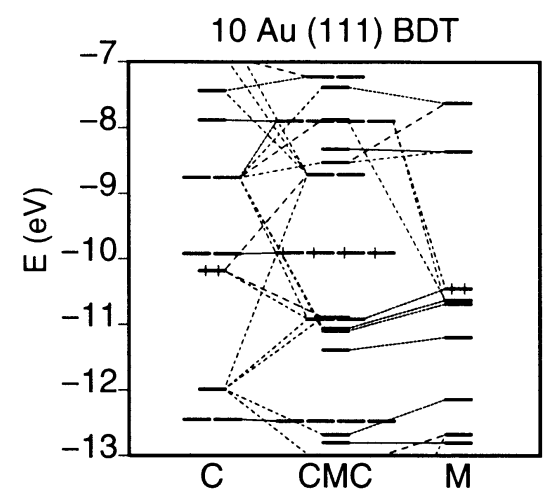

(b)

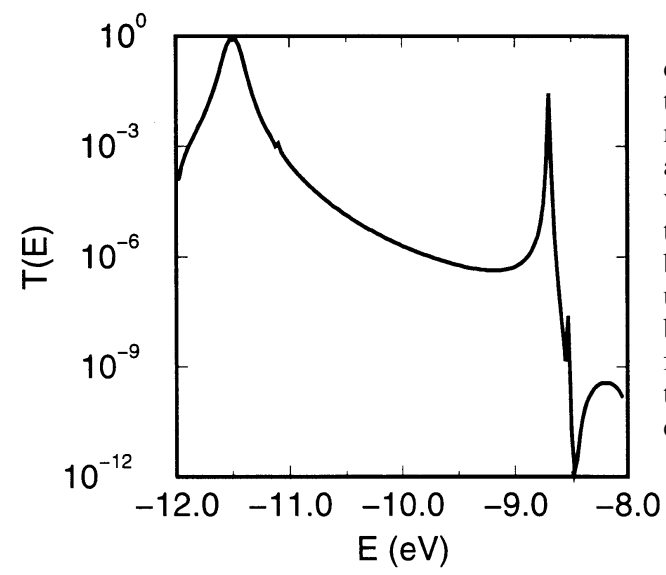

FIGURE 4. (a) Energy-level diagram for $10 \mathrm{Au}$ (111) cluster and BDT system. (b) Transmission vs. electron energy $\mathrm{E}$, and (c) conductance vs. bias voltage $\mathrm{V}$, for this system. In the energy-level diagram, $\mathrm{C}$ labels the energy levels of the uncoupled left cluster, M labels the energy levels for the free molecule, and CMC labels the energy levels for the bonded clusters + molecule system.

(c)

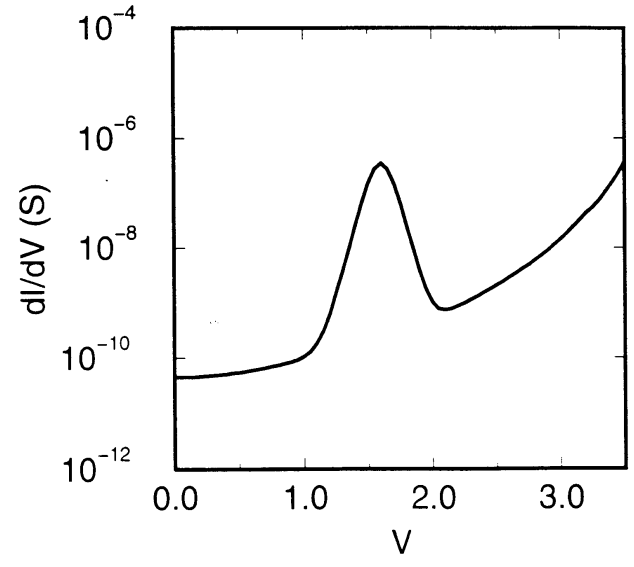


(a)

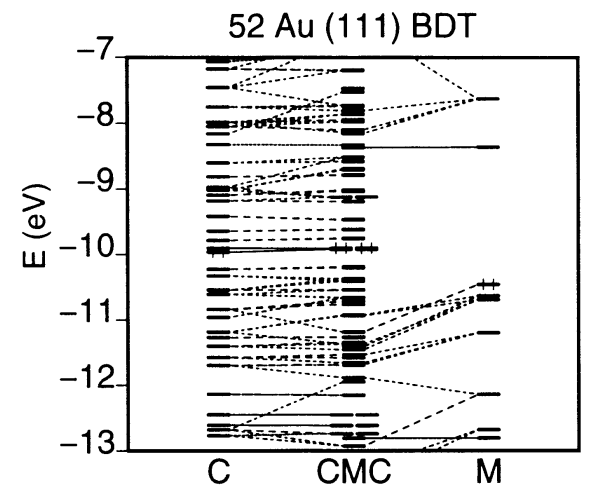

(b)

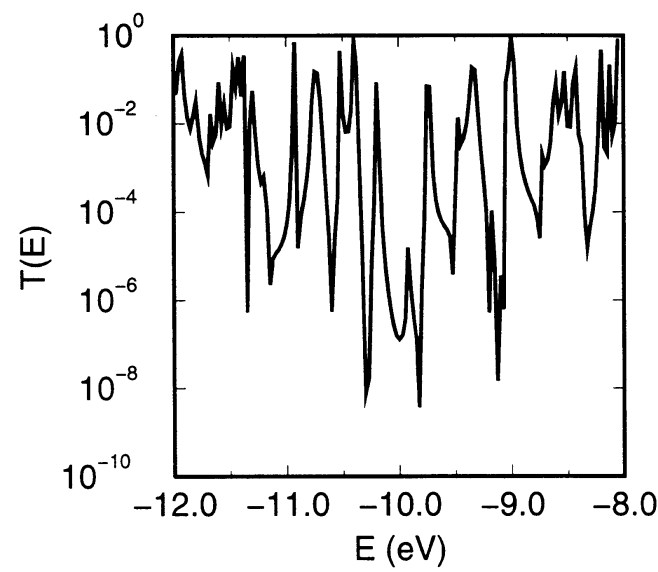

Figure 5. (a) Energy-level diagram for $52 \mathrm{Au}$ (111) cluster and BDT system. (b) Transmission vs. electron energy $\mathrm{E}$, and (c) conductance vs. bias voltage $\mathrm{V}$, for this system.

(c)

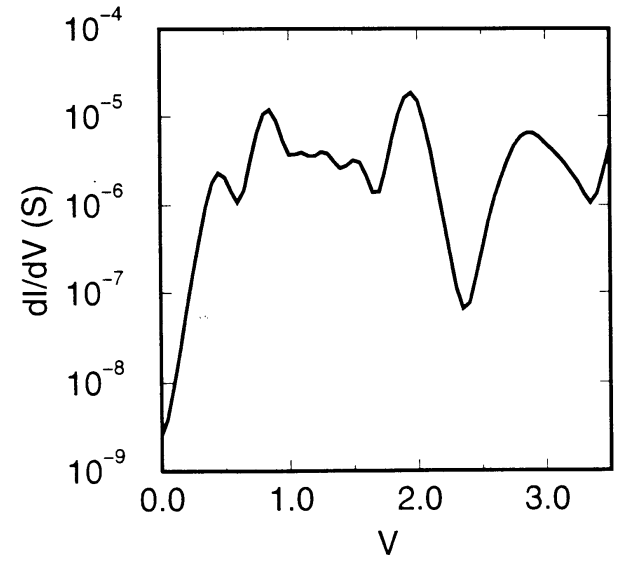


(a)

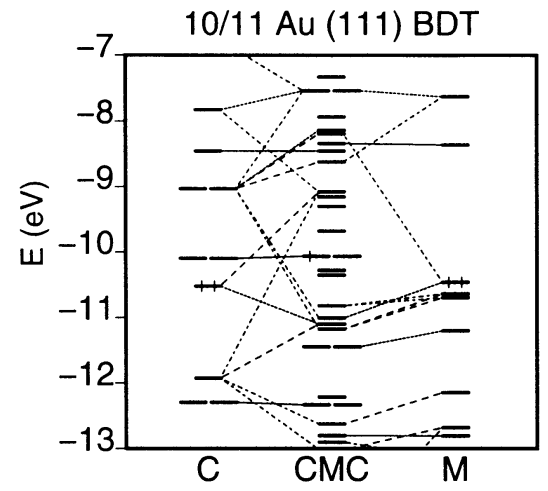

(b)

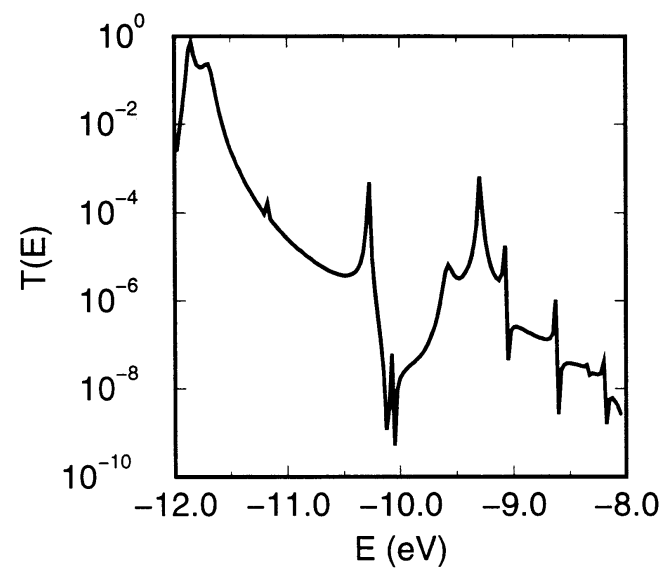

Figure 6. (a) Energy-level diagram for 10/11 Au (111) clusters and BDT system. (b) Transmission vs. electron energy $\mathrm{E}$, and (c) conductance vs. bias voltage $\mathrm{V}$, for this system.

(c)

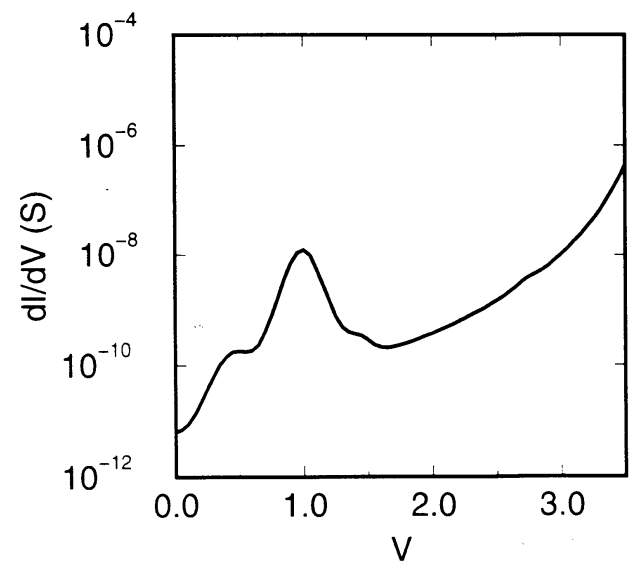


For the clusters of $10 \mathrm{Au}$ atoms (FIGURE 3a) it is seen in the energy spectrum (FIGURE 4a) that the CMC system is a mixture of molecular and cluster states. The BDT levels around its HOMO, which have large S content, mix into different levels of the combined system. The Fermi level for the cluster (approximated by the HOMO for the cluster) lies in the HOMO-LUMO gap of the molecule, and this has the effect of narrowing the gap in the combined system. Other geometries with similar numbers of atoms have also been studied, but are not presented here. The same general effects are noticed; the addition of the Au cluster alters the molecular states. It seems that the relevance of the BDT HOMO-LUMO to the combined system is questionable due to the mixing of states that occurs. Transport in such a system will be different from that through a free molecule.

The disadvantage of modeling with small clusters is that they do not fully capture the continuum character of the leads. Considerably bigger clusters were constructed in order to generate a denser spectrum. These larger clusters were simplified by using only 6 s orbitals on most of the Au atoms. Only the Au atoms neighboring the molecule were modeled using all of the valence orbitals for Au. This is different from the smaller clusters, where all cluster atoms were modeled using the full valence orbital set. The incident electrons are from the $6 \mathrm{~s}$ band, and although it is known that the $5 \mathrm{~d}$ and $6 \mathrm{p}$ orbitals play a role in shifting the 6 s energies, the inclusion of only $6 \mathrm{~s}$ makes the calculation for large clusters feasible.

In the energy level diagram given in FIGURE 5a for the cluster consisting of $52 \mathrm{Au}$ atoms (FIGURE 3c), the levels around the HOMO are predominantly shifted further below the Fermi energy, and the levels around the LUMO are mixed into levels that still lie above the Fermi energy. It is possible to argue that there is still a BDT HOMO-LUMO character to the resulting combined system, in that there is a gap between where most of the HOMO levels mix and where the LUMO levels mix. In the latter sections of this paper the usefulness of this notion will be seen to be limited since it will be shown that there can be transport through levels not connected with the molecule's HOMO or LUMO.

The other configuration to be presented is that of asymmetric binding - FIGURE $3 \mathrm{~b}$. It is reasonable to assume that the left part of the break junction will not be a mirror image of the right part. This is modeled by considering a CMC system with two different clusters. In the energy-level diagram for this system (FIGURE 6a) degeneracies are lifted, and as a result the levels are more uniformly distributed than in the case of two $10 \mathrm{Au}$ clusters. The energy levels, to which neither the left cluster nor molecule connect are due to the right cluster.

It should be pointed out that all the energy diagrams presented have been calculated with the first atom of both ideal leads attached to the CMC. The lead atom is modeled using an Au 6s orbital. This coupling, W, can shift and split the energy levels. It has been found, though not shown here, that it is possible to attach the ideal leads in such a way that the energy spectrum changes quite significantly. Here the leads are attached on the central axis so as not to break the symmetry of the CMC, thereby preserving the general energy structure of the CMC. 
(a)

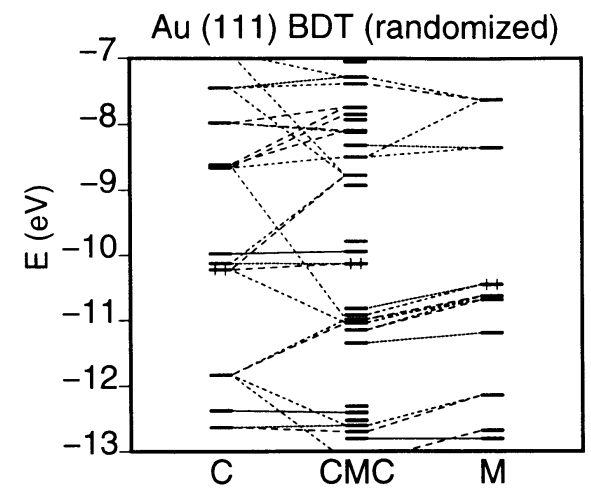

(b)

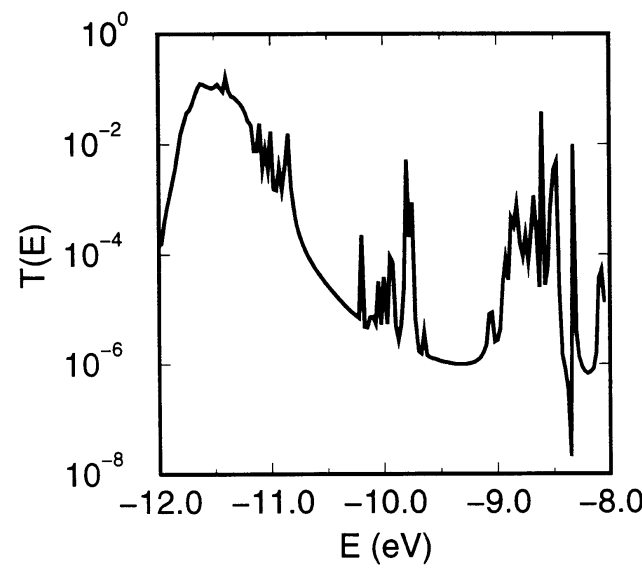

FIGURE 7. (a) Energy-level diagram for $10 \mathrm{Au}$ (111) cluster and BDT system for randomized cluster geometry. (b) Averaged transmission vs. electron energy $\mathrm{E}$, and (c) conductance vs. bias voltage $\mathrm{V}$, for this system.

(c)

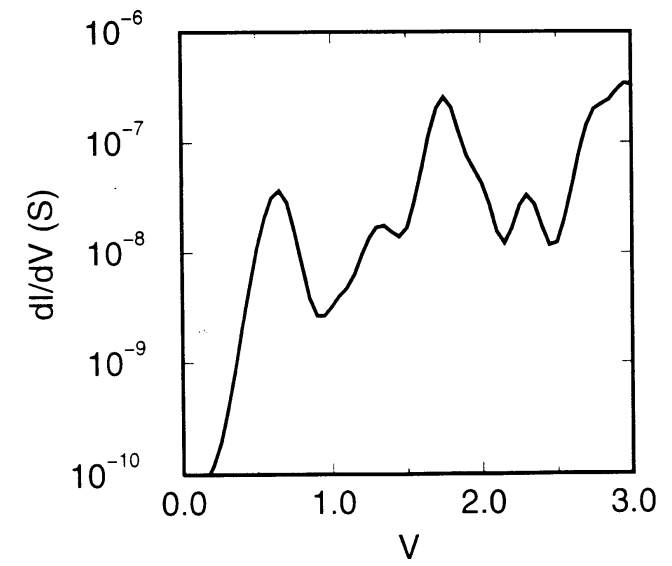




\section{TRANSMISSION AND CONDUCTANCE OF CMC SYSTEMS}

Using the LS formalism, the transmission through the above systems was calculated for energies around the Fermi level of the Au leads. The Landauer formula was then used to evaluate the current vs. bias voltage curve for each system. The differential conductance was calculated from this data.

The transmission curve for the $10 \mathrm{Au}$ cluster is shown in FIGURE $4 \mathrm{~b}$. It has a region of strong transmission below the Fermi energy, and peaks of weaker strength above. The transmission at $-11.5 \mathrm{eV}$ can be attributed to a $\mathrm{S} \pi-$ and $\mathrm{C} \pi$-like state with some Au presence. The little peak at $-11.1 \mathrm{eV}$ is due to a CMC $\sigma$-like state. The transmission at $-8.7 \mathrm{eV}$ is due to a $\sigma$ like state also. The smaller peak at $-8.52 \mathrm{eV}$ is due to the BDT LUMO which has been mixed with some cluster states. Nonetheless, the LUMO for the CMC is different from the LUMO for the BDT. It was mentioned previously that the coupling of the lead has the effect of shifting the energy levels of the CMC. The coupling of the entire lead to the CMC can further shift the levels, which is the case for the peak at $-11.5 \mathrm{eV}$. This is due to the state at around $-11.4 \mathrm{eV}$ in the spectrum, which has strong binding to the lead.

The corresponding conductance curve is shown in FIGURE 4c. It has been calculated with a lead Fermi energy at $-9.5 \mathrm{eV}$, which seems reasonable based on the energy level diagram presented. The lack of any transmission peak around the Fermi energy, leads to the valley around 0 applied bias. The two peaks at -11.5 and -8.7 , and their spacing give rise to the structure in the conductance.

For the $52 \mathrm{Au}$ cluster, there are many more CMC levels to tunnel through. One would expect though that only those levels that have a wavefunction which extends across the whole of the system would be conducting. This is found. There is transmission at energies which fall within the HOMO-LUMO gap of the molecule, and for these states there is enough presence on the molecule to transmit (see FIGURE $5 b)$. These levels, though they have $\pi$ character, are not connected strongly to either the HOMO or LUMO of the BDT, and yet still conduct. Though these levels have molecular content, it is not possible, as is shown in the energy diagram, to attribute this content to any particular molecular orbital. These states seem to be admixtures of many states from the molecule.

Because of the transmission over virtually all of the energy range, the corresponding conductance curve, FIGURE 5c, has much more structure. The Fermi energy was chosen to be $-9.98 \mathrm{eV}$ since for a larger cluster the HOMO of the cluster should approximate the Fermi level. Because there are many more peaks in the transmission spectra, the magnitude of the differential conductance has risen. The corresponding rise in the conductance also occurs at a voltage that is closer to 0 than what was found experimentally. Other theoretical work by Kemp et al. and Datta et al. ${ }^{12}$ has also found that the calculated conductance for BDT to be greater than what was experimentally measured.

Finally, the last case to be considered is for the molecule binding to two dissimilar clusters. Notice that in the conductance curve (FIGURE 6c) the magnitude is down compared to the other two configurations. In the transmission diagram (FIGURE 6b) the common characteristic of the larger peaks is that the wavefunction of the CMC has significant presence on the cluster. The smaller peaks are due to states that are more localized on the molecule. The major transmission at $-11.6 \mathrm{eV}$ is due 
to predominantly $\sigma$ bonds between the cluster and molecule. This state is also highly coupled to the lead. The same can be said about the peak at $-9.3 \mathrm{eV}$, where the state is again $\sigma$ like, with good coupling to the lead, though not as strong as the one at $-11.6 \mathrm{eV}$.

The conductance curve is shown in FIGURE 6c. The Fermi energy was chosen to be $-9.8 \mathrm{eV}$. With the two transmission peaks at $-10.4 \mathrm{eV}$ and $-9.3 \mathrm{eV}$, the conductance rises at around $1 \mathrm{~V}$. The large transmission peak at $-11.6 \mathrm{eV}$ contributes to the rise in the tail of the conductance curve at $3 \mathrm{~V}$.

\section{FINITE TEMPERATURE EFFECTS}

The above calculations for the conductance have included some finite temperature effects by using the $300 \mathrm{~K}$ Fermi distribution for the source and sink electrons via the Landauer formula. However the CMC system has been assumed to be in a regular geometrical configuration. At room temperature the effects of thermal vibration on the configuration of the cluster will be significant. A simplified way to model these effects, ignoring inelastic scattering, is to examine the nature of positional disorder in the Au clusters at room temperature. This model does not seem unreasonable, since the gold clusters do not form a perfect lattice, and hence deviations from the regular cluster geometry could be important.

EXAFS measurements on small clusters have given values for the Debye-Waller factor, ${ }^{20}$ which can be used to obtain the rms displacement of atoms in a solid at a temperature $T$. Different geometrical configurations of a given cluster were generated by randomly displacing each Au atom in the cluster according to a Gaussian distribution consistent with this value of the rms displacement. For each random configuration of the various clusters, the transmission was evaluated. FIGURE 7 shows the effect of randomizing the positions on the energy spectrum, the transmission, and the conductance. The transmission is an average of over 50 random configurations of the $10 \mathrm{Au}$ clusters. All the energy-level degeneracies that exist in the regular geometry are broken due to the positional change. It is evident that this causes a general broadening in the transmission spectra, as peaks are shifted or new ones created due to the change in geometry. The transmission is also weaker than in the regular geometry. This smearing effect causes the conductance peaks to broaden, and in some cases flatten into plateaus as shown in FIGURE 7c. A sampling of only a few random configurations can not be expected to completely describe the physical situation, but it can be seen that the effect of geometric disorder does play a significant role.

\section{CONCLUSION}

We have presented calculations for the room-temperature differential conductance of BDT. The role of the contacts has been studied by including them with the molecule in the quantum chemistry calculation at the extended Hückel level. This has shown that there is mixing between cluster and molecule states, which alters the energy levels of the free molecule. It has been found that the resulting transport and 
conductance is sensitive to the geometry of the Au cluster contacts. This is because the electronic structure of the system is sensitive to the CMC configuration.

It was also found that the size of the clusters has noticeable effects on the conductance. With the larger size, a denser spectrum of cluster states is achieved, which is a better approximation to the continuum of states that would exist in reality. Thus many CMC states occur between the BDT HOMO and LUMO, and many of these are transmitting. This leads to a rise in conductance at smaller voltages than were observed experimentally. The magnitude is also up by a few orders compared to experiment. Thus experimentally, the molecule is less transparent than what our theory would seem to suggest. Why this is the case is still an open question.

To include more finite temperature effects in the model, positional disorder within the gold clusters was considered. By changing the regular geometry of the cluster, the energy levels were shifted around, thereby moving or creating new transmission peaks. The effect of averaging over configurations broadened and lowered the magnitude of the transmission spectrum. The conductance was also broadened and flattened in some regions. This appears to be consistent with the experimental findings, where variations in the conductance are relatively smooth and less pronounced. Thus positional disorder does seem to be a factor that has a role in determining the experimentally observed conductance.

\section{ACKNOWLEDGMENTS}

We would like to thank Mark Reed, Ross Hill, and John Black for helpful discussions. For the extended Hückel calculations, we would like to acknowledge the EHC program, Yaehmop, by Greg Landrum. This work was supported by NSERC.

\section{REFERENCES}

1. Aviram, A. \& M.A. Ratner. 1974. Molecular Rectifiers. Chem. Phys. Lett. 29: 257.

2. Reed, M. A., C. Zhou, C. J. Muller, T. P. Burgin \& J. M Tour. 1997. Conductance of a molecular junction. Science 278: 252-253.

3. Zhou, C., M. R. DeshPande, \& M. A. ReED. 1997. Nanoscale metal/self-assembled monolayer/metal heterostructures. Appl. Phys. Lett. 71: 611.

4. Stipe, B.C., M.A. Rezaei, W. Ho, S. GaO, M. Persson \& B.I. Lundevist. 1997. Single-molecule dissociation by tunneling electrons. Phys. Rev. Lett. 78: 4410-4413.

5. AndRes, R.P., J.D. Bielefeld, J.I. Henderson, D. B. Janes, V.R. Kolagunta, C.P. KUbiaK, W.J. MAHONEY \& R.G. OsIfChIN. 1996. Self-assembly of a two-dimensional superlattice of molecularly linked metal clusters. Science 273: 1690-1693.

6. Mirkin, C. A., R. L. Letsinger, R.C. Mucic \& J.J. Storhoff. 1996. A DNA-based method for rationally assembling nanoparticles into macroscopic materials. Nature 382: 607-609.

7. Fischer, C.M., M. Burghard, S. Roth \& K. von KlitZing. 1995. Novel tunneling experiments on organic heterostructures. Surf. Sci. 361/362: 905-908.

8. Datta, S., W.Tian, S. Hong, R. Reifenberger, J.I. Henderson \& C.P. Kubiak. 1997. Current-voltage characteristics of self-assembled monolayers by scanning tunneling microscopy. Phys. Rev. Lett. 79: 2530-2531. 
9. Magoga, M. \& C. JoAChim. 1997. Conductance and transparence of long molecular wires. Phys. Rev. B 56: 4722-4729.

10. Datta, S. \& W.Tian. 1997. Application of the Friedel sum rule to symmetric molecular conductors. Phys. Rev. B 55: R1914-R1917.

11. JOACHim, C., \& J.F. VinUESA. 1996. Length dependence of the electronic transparence (conductance) of a molecular wire. Europhys. Lett. 33: 635-640.

12. KeMP, M et al. \& S. DATTA et al. 1997. Molecular Electronics - Science and Technology Conference. Unpublished material.

13. Mujica, V., M. Kemp, A. Roitberg \& M. Ratner. 1996. Current-Voltage characteristics of molecular wires: Eigenvalue staircase, Coulomb blockade, and rectification. J. Chem. Phys. 104: 7296-7305.

14. Samanta, M.P., W. Tian, S. Datta, J.I. Henderson, \& C.P. Kubiak. 1996. Electronic conduction through organic molecules. Phys. Rev. B 53: R7626-R7629.

15. Kemp, M., A. Roitberg, V. Mujica, T. Wanta \& M. A. Ratner. 1996. Molecular Wires: Extended Coupling and Disorder Effects. J. Phys. Chem 100: 8349-8355.

16. Sellers, H., A. Ulman, Y. Schnidman \& J.E. Eilers. 1993. Structure and binding of alkanethiolates on gold and silver surfaces: implications for self-assembled monolayers. J. Am. Chem. Soc. 115: 9389-9401.

17. Laibinis, P.E., G.M. Whitesides, D.L. Allara, Y.T. TaO, A.N. Parikh, R.G. NuZzo. 1991. J. Am. Chem. Soc. 113: 7152.

18. Correia, A. \& N. GarCiA. 1996. Nanocontact and nanowire formation between macroscopic metallic contacts observed by scanning and transmission electron microscopy. Phys. Rev. B 55: 6689-6692.

19. Kondo, Y. \& K. TAKAYANAGI. 1997. Gold nanobridge stabilized by surface structure. Phys. Rev. Lett. 79: 3455-3458.

20. Pinto, A., A. R. Pennisi, G. Faraci, G. D'Agostino, S. Mobilio \& F. BOSCHERINI. 1995. Evidence for truncated octahedral structures in supported gold clusters. Phys. Rev. B 51: 5315-5321. 